\title{
Myoelectric Prosthetic Hand with Air Muscles
}

\author{
Jibin Jose, Rosemary James, Meera Alex, Mithu Saseendran, Anu Johnson and Ashish Sundar
}

\begin{abstract}
Prosthesis development were historically been done in order to find a matching replacement for human hand which is unique in exhibiting its dexterous properties. Prosthesis controls that are established with the help of heavy motors make it a very complex actuation mechanism with relatively less power and efficiency. This paper describes an EMG - activated, tendon - driven, air muscle mechanism which delivers, relatively high power to weight ratio with higher efficiency by mimicking human muscle motion. The EMG signal is acquired from the skin surface by surface EMG electrodes and are conditioned using the basic techniques. Using spring loaded mechanism the weight of the hand prosthesis is kept to a minimum. Air muscles are used in for better control and to improve power to weight ratio. The flexion movements are achieved with a nylon string (tendon).
\end{abstract}

Keywords--- Myoelectric hand, tendon driven mechanism, air muscles, endoskeleton

\section{INTRODUCTION}

$\mathrm{T}$ HE history of prosthesis development dates back to a period when the medical thoughts began; at around 300 B.C with wooden and copper replacements [1] . Amputation is the removal of anybody extremities which may be due to trauma, surgery or relieving pain. Prostheses were developed as an aid to the amputees which will meet either functional or cosmetic needs. The need for prosthesis has increased as the rate of people being amputated is relatively high every year. Although many companies and research works have been done for developing prostheses, it is a difficult task for creating prostheses that can meet both functional and cosmetic needs of amputees. Prostheses can be broadly classified into two endoskeleton and exoskeleton prosthesis. The exoskeleton prostheses are found to be cosmetically much better than the endoskeleton ones and the range of motion is limited to a great extent, whereas the endoskeleton prostheses are functionally better and offers higher degrees of freedom. The various parameters of concern while designing prostheses are its functionality, cosmetic capability, weight and cost. Most hand prosthesis available is electric motor actuated prosthesis. The

Jibin Jose, Student, Biomedical Engg., Sahrdaya College of Engg. \& Tech., Thrissur, India.E-mail:jibin718@gmail.com

Rosemary James, Student, Biomedical Engg., Sahrdaya College of

Engg. \& Tech., Thrissur, India.E-mail:rosemoljamest@gmail.com Meera Alex, Student, Biomedical Engg., Sahrdaya College of Engg. \& Tech., Thrissur, India.E-mail:meera.sahrdaya@gmail.com

Mithu Saseendran, Biomedical Engg, Sahrdaya College of Engg.\& Tech,Thrissur,India.E-mail:gs2512848@gmail.com Anu Johnson, Biomedical Engg., Sahrdaya College of Engg.\& Tech,Thrissur,India.E-mail:anujhonson1994@gmail.com

Ashish Sundar, Assistant Professor, Biomedical Engg., Sahrdaya College of Engg. \& Tech., Thrissur, India.E-mail:ashishsundar@gmail.com use of motor makes it complex and heavy. Thus reducing the complexity in the design of prosthesis without compromising the power delivering efficiency should be a major concern while designing a prosthetic limb. This paper focuses on developing a myoelectric prosthetic hand with increased power/weight ratio by using pneumatic - controlled tendon driven muscle actuation mechanism. This was designed by developing an air muscle which can be controlled by pneumatic valves [2]. The air muscle action is coupled into a tendon attached to it with the help of single pulley interconnection across the muscle which is then coupled to the three fingered hand via a spring loaded system placed at the metacarpal inter phalanges and proximal inter phalanges joints [3]. The electromyogram (EMG) signals generated from the residual limb of the amputee can be used for triggering the actuation. This is a simple yet powerful method that can be employed in developing prosthesis, biotech labs assistance or can be mounted in wheel chairs for the elderly people.

\section{METHODS AND DESIGN METHODOLOGIES}

The electrical potential developed when muscle fibers contract is known as Electromyogram or EMG. A typical EMG signal that is generated within the body will be having duration of about 3-15 ms, with amplitude values ranging from 20-2000 $\mu \mathrm{V}[3]$.

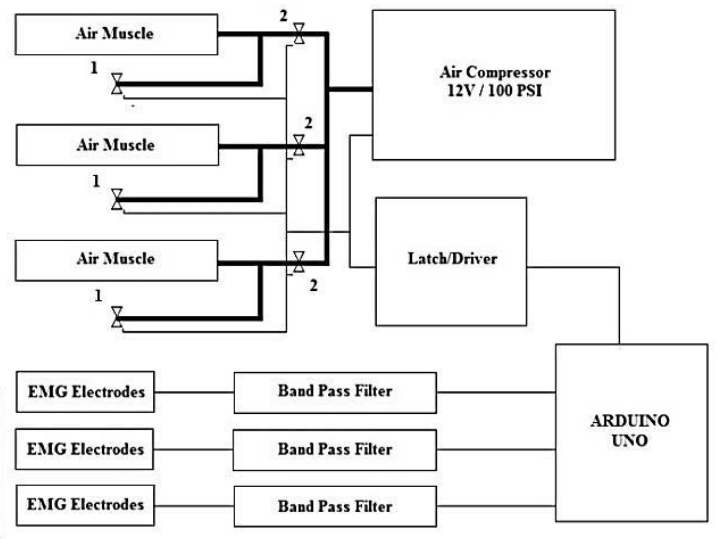

Figure 1: System block diagram

The typical energy range of EMG signals are between 50 500 Hz. EMG signals are conditioned using an instrumentation amplifier section, filter and fed into microcontroller. The microcontroller reads these signals and makes decisions based on the algorithm to control the pneumatic valve which in turn drives the prosthesis. Thus the whole setup can be divided into three sections; A) Electromyogram B) Microcontroller and pneumatic control C) Prosthetic design. The Fig.1 shows the block diagram description of the myoelectric hand prosthesis. As shown in block diagram the complete system is microcontroller based 
EMG activated prosthesis for upper limb amputees.

\section{A. Electromyogram}

Bipolar acquisition was employed using disposable surface ECG electrodes to acquire EMG from the residual limb of the subject and coaxial cables were used as the leads due to their noise reduction shielding. The quality of the signals depends on the placement of the electrodes and the size of the residual muscle. The Texas Instruments INA-118P instrumentation amplifier is used for its very high common-mode rejection ratio (CMRR) of about 120dB.The Fig.2 shows the image of the acquired EMG signal.

$$
G=1+\left(\frac{50 K \Omega}{R g}\right)
$$

$G$ is the gain

$R g$ is the resistance across $1^{\text {st }}$ and $8^{\text {th }}$ pin of INA 118

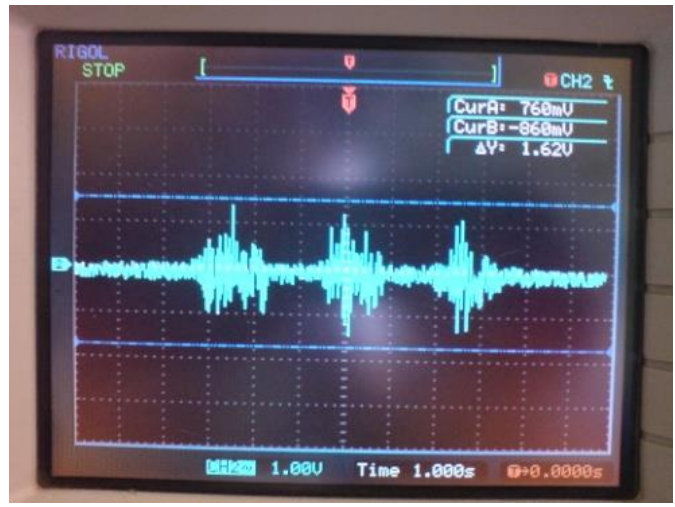

Figure 2: Acquired EMG signal

A fourth order, Bessel's band pass filter is selected for its linear phase - frequency response. The band pass filter selected for this application is Bessel's fourth order filter. Filter parameters are given in Table 1.

Design of high pass filter:

$$
F_{c}=\frac{f_{n}}{2 \pi \mathrm{RC}}
$$

Design of low pass filter:

$$
F_{c}=\frac{1}{2 \pi f_{n} \mathrm{RC}}
$$

$F_{C}$ is the cut-off frequency (in $\mathrm{Hz}$ ), $f_{n}$ is the function for Bessel's filter

$\mathrm{R}$ and $\mathrm{C}$ are the resistors and capacitors

Table 1: Filter Parameters

\begin{tabular}{|l|c|c|c|c|c|}
\hline $\begin{array}{c}\text { Filter } \\
\text { Order }\end{array}$ & $\begin{array}{c}\text { Butter- } \\
\text { worth }\end{array}$ & \multicolumn{2}{|c|}{ Bessel } & \multicolumn{2}{c|}{ Chebyshev } \\
\hline & K(gain) & $f n$ & K(gain) & $f n$ & K(gain) \\
\hline 2 & 1.586 & 1.272 & 1.268 & 1.231 & 1.842 \\
\hline 4 & 1.152 & 1.432 & 1.084 & 0.597 & 1.582 \\
& 2.235 & 1.606 & 1.759 & 1.031 & 2.660 \\
\hline 6 & 1.068 & 1.607 & 1.040 & 0.396 & 1.537 \\
& 1.586 & 1.692 & 1.364 & 0.768 & 2.448 \\
& 2.483 & 1.908 & 2.023 & 1.011 & 2.846 \\
\hline
\end{tabular}

\section{B. Microcontroller}

Myoelectric hand prosthesis described in this paper is activated by a microcontroller based system. In most of the cases upper limb amputees have EMG signals rising from the residual muscles which may be of $100 \mu \mathrm{V}$ amplitude [4].

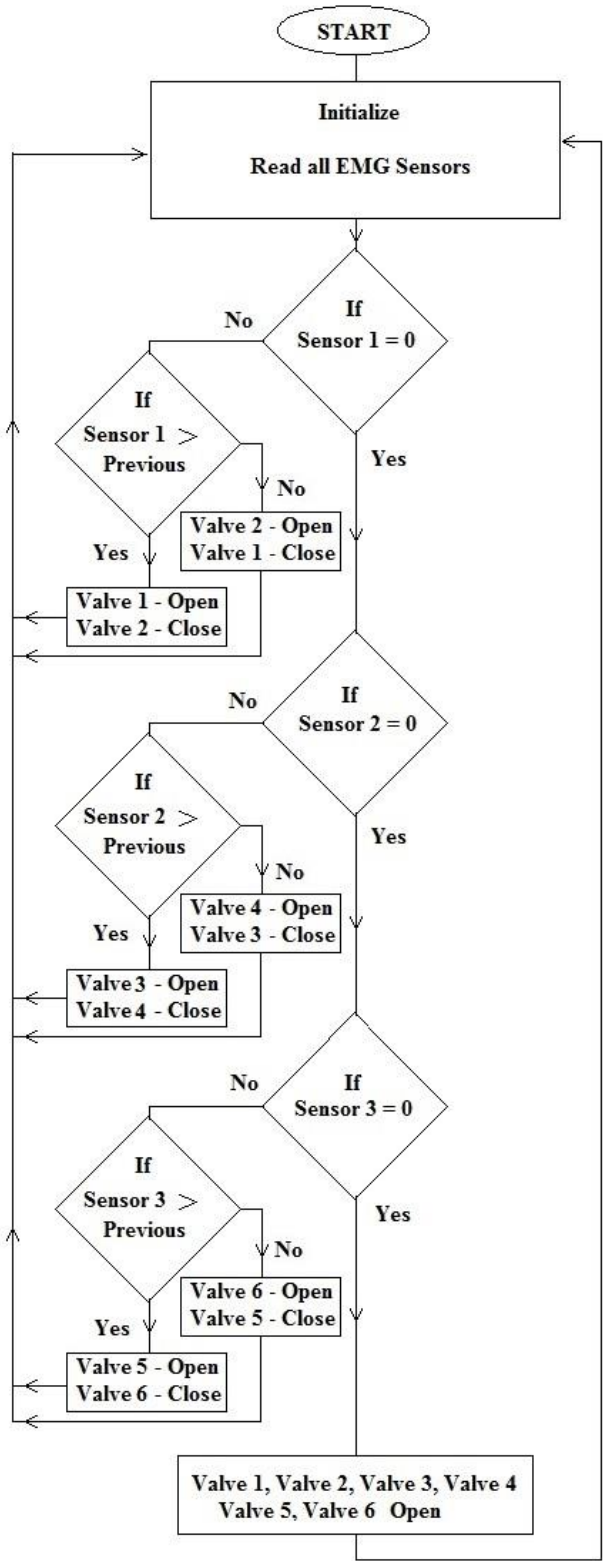

Figure 3: Flow chart

The main objective is the processing of the EMG signals from the residual muscles and controlling the movement of the mechanical parts with the help of air muscle actuation. Upon receiving the EMG signal, microcontroller runs its initializing operation. Then when a valid signal is available the controller checks for the action to be performed. The Fig. 3 shows the flow chart for the proposed system. This gives the necessary control for the valve operation and muscle action.

Arduino is a single board microcontroller designed to provide an ease of access environment suitable for various 
applications. It is an open source hardware board consisting of ATmega 328 processor which is pre-programmed with a boot loader that avoids external loading of program into the microcontroller memory. It has 32 Kilobytes flash memory and 14 digital $\mathrm{I} / \mathrm{O}$ pins with an operating voltage of $5 \mathrm{~V}$. These boards are implemented using USB, with USB to serial convertor FT232 or 16U2. The arduino output pins 0 , $1,9,10,11,12,13$ are used for valve controls in the three fingered hand prosthesis. The fig. 4 shows the hardware of the implemented system. The Arduino is interfaced with LCD to show activation, initialization, reading, deflation and inflation according to the corresponding EMG inputs.

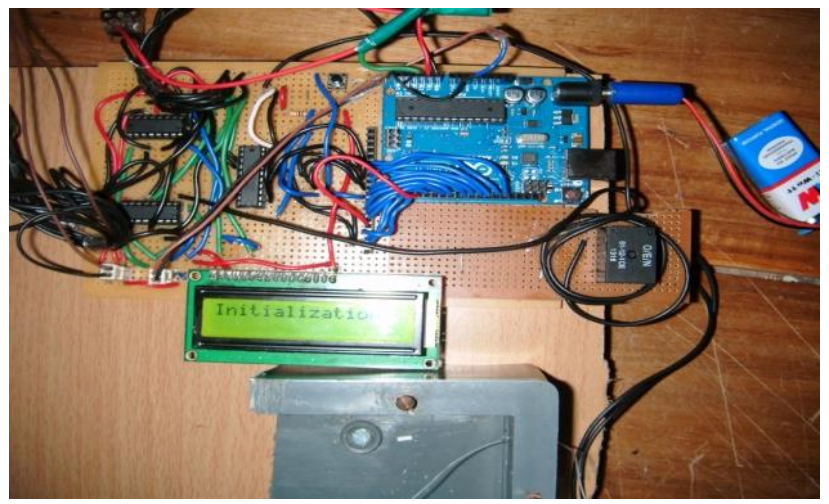

Figure 4: Hardware

\section{Air Muscle and Prosthetic Design}

The key element of this work is the design of air muscle that mimics the biological muscle counter action. A trial and error analysis has been done before deciding the material suitable for muscle replica. The first trial was done on the pure silicon tube which showed no change for whatever pressure being applied. The next trial was done on the tourniquet tube which expanded well but exploded soon after applying a pressure of 20 PSI.

Chart 1: Material analysis

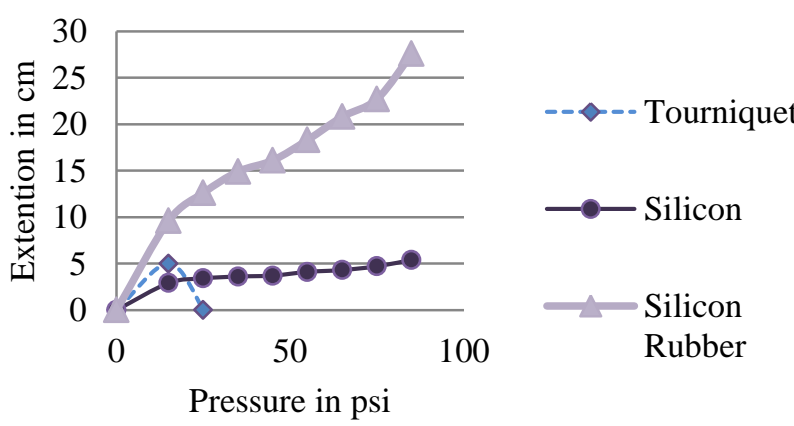

Finally silicon rubber tube which has high elastomeric properties was taken and this material exhibited relatively good expansion by applying a pressure in the range of 40-60 PSI. The Chart 1 shows a graphical representation of material analysis performed on three different types of materials. Air Muscle is an actuator that is works in a similar manner to a human muscle with combined characteristics of smoothness and compliance to deliver power with accurate motion control ability. The silicon rubber tube air muscle is responsible for the flexion and extension of the hand. To prevent the rupture of air muscle, as a precaution a nylon cloth sleeve was put over the tube. The Figure 5 and 6 shows the comparison between the air muscle and a ten rupee note which is $13.5 \mathrm{~cm}$ long, the relaxed and extended muscle is shown in the figure. On applying a pressure of 40-60 PSI the muscle extended upto $19.5 \mathrm{~cm}$. The results have shown that for 1 PSI there is extension of $3 \mathrm{~mm}$ in length for tube.

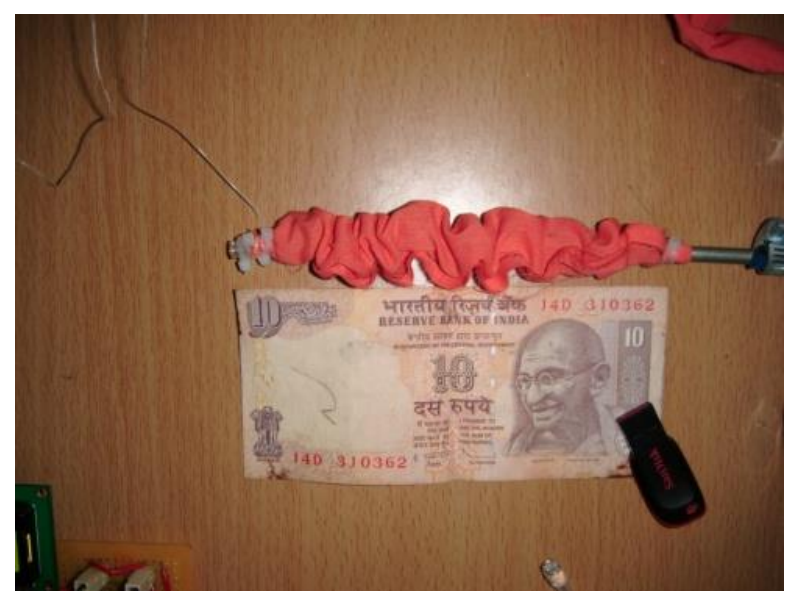

Figure 5: Relaxed Muscle

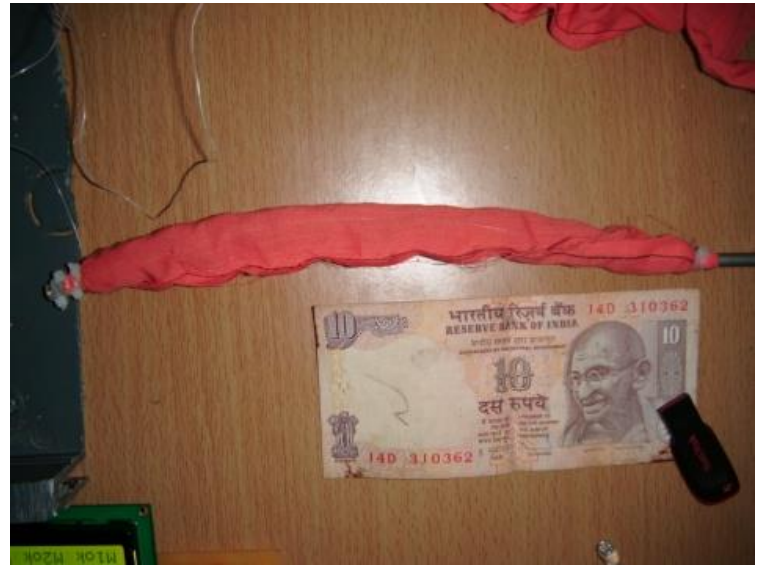

Figure 6: Extended Muscle

The prosthesis design has been done in order to make joints similar to the human hand [4] .Human hand is made up of finger bones called phalanges, they are present at each finger enabling us to perform smooth action. This project has been designed with the MIP and PIP phalange joints. The photograph of the prosthesis is shown Fig. 7, which is a three fingered system consisting of 6 joints and 9 bone segments

The prosthesis is made with plastic rod attached with spring loaded tendon driven system for the finger extension and flexion. This model has metacarpointerphalangeal and proximal interphalangeal joints made by attaching hinges at the joint interfaces. The prosthesis control is done with the help of tendon driven mechanism; basically two types of motion are done in this model; extension and flexion [5]. On actuation of the air muscle, the tendon drives the prosthesis placed at 110 degrees to undergo a flexion angle of 85 degrees and once the air is released the spring pulls the hand back to 
its normal position. Hardware is programmed to sense the sensor inputs and establish necessary control of hand extension and flexion.

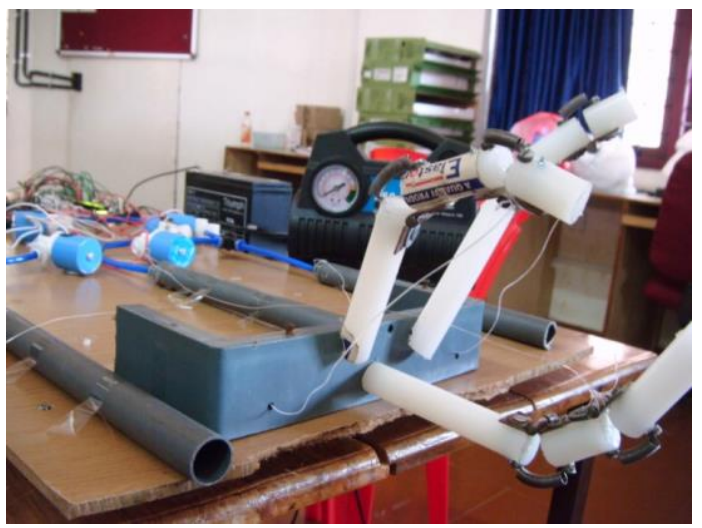

Figure 7: Myoelectric hand Prosthesis

\section{RESULT AND CONCLUSION}

The Myoelectric hand prosthesis is a three fingered system that is actuated by pneumatic controlled tendon driven air muscle to produce two types of motion flexion and extension. The maximum flexion angle was found to be 85 degrees whereas the maximum extension angle is of 110 degrees .The model exhibited an extension of $3 \mathrm{~mm}$ for 1PSI pressure with a flexion angle of 0.5 degrees . The air muscle (silicon rubber) is the key functional element in this prosthesis, which on application of 40-60 PSI pressure extended fifty percent more the original length. Thus the system has increased the functional efficiency of the prosthesis by delivering high power to weight ratio.

The tendon driven spring loaded myoelectric hand prosthesis a new mechanism of prosthesis development and control. This proposal could be useful in increasing the functional efficiency of prosthesis without compromising other contributing factors; also this mechanism is useful in production of cost effective and powerful prosthesis development. This prosthesis has been designed to perform grasp and hold application. Also by increasing the number of air muscles the power delivered can be made increased to a greater extent and there increase the number of degrees of freedom.

\section{REFERENCES}

[1] Laurein.L.Smith,"ProstheticLimbDevelopment:AHistoricalReview" ,Case Undergraduate Research Journal ,Volume 1,2006

[2] Ranjeeth Rajan, Dr.P.K.Upadhyay, Dr. Arbind Kumar, Dr. Praveen Dhyani, "Theoretical and Experimental Modelling of Air muscle", International Journal of Emerging Technology and Advanced Engineering ,Volume 2, Issue 4, April 2012

[3] W.Ganong, "Review of Medical Physiology", $20^{\text {th }}$ ed.NewYork,Mc Graw.Hill,2001,PP.72-74

[4] Ton-Tai Paan,Ping-LinFan,HuihuaKennyChiang,Rong-Seng chang,and Joe-AirJiang,Mechatronic Experiments Course Design:A Myoelectric controlled Partial Hand Prosthesis,ieee transanctions education,Vol.47,no.3, august,2004

[5] Rajiv Doshi, Clement Yeh, Maurice LeBlanc: The design and development of a gloveless endoskeletal prosthetic hand, Journal of Rehabilitation Research and Development, Vol. 35 No. 4. Oct 1998, Pages 388 - 395 